\title{
CO-JP-1
}

\section{Ground structure approach for PZT layout optimization in semi-active vibration control systems of space structures}

\author{
Akihiro Takezawa*, Kanjuro Makihara**, Nozomu Kogiso***, Mitsuru Kitamura* \\ *Department of Mechanical System and Applied Mechanics, Graduate School of Engineering, Hiroshima University, \\ 1-4-1 Kagamiyama, Higashi-Hiroshima, Hiroshima, Japan \\ E-mail: akihiro@hiroshima-u.ac.jp \\ * Department of Aerospace Engineering, Graduate School of Engineering, Tohoku University, \\ 6-6-04, Aramaki Aza Aoba Aoba-ku, Sendai, Miyagi, Japan

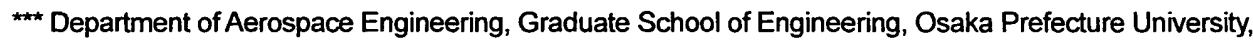 \\ 1-1 Gakuen-Cho, Naka-Ku, Sakai, Osaka, Japan
}

\begin{abstract}
In this research, an optimization methodology is proposed for the piezoelectric transducer layout and length of energy-recycling type semi-active vibration control system for a space structure. The design variables are set as the length of the piezoelectric actuator on each truss element. Physical properties of the actuator are formulated as the function of the design variables. The vibration analysis problem of the truss structure is formulated based on the transient response analysis using the modal method. The objective function is set as the integration of the square of all displacement over the whole analysis time domain. The sensitivity of the objective function is derived based on the adjoint variable method. Based on these formulations, an optimization algorithm is constructed using the method of moving asymptotes (MMA). Numerical examples are provided to illustrate the validity and utility of the proposed methodology.
\end{abstract}

Key words: Semi-active vibration control, Piezoelectric transducer, Truss structure, Optimization

\section{Introduction}

Vibration suppression is one of the most important subjects in designs and operations of space structures. Due to the launch limit of the rocket, such structure might have extremely light weight and it could lead to the small stiffness which is easily vibrated. Moreover, different from the earth, vibration energies are hard to be scattered in the space vacuum environment. These problems could be overcome by vibration control methodologies. A large number of researches on synthesis of piezoelectric transducer (PZT) and electric devices, which is the most major structural vibration suppression methodology, were performed $[1,2]$. PZTs attached to or embedded in structures can convert mechanical energy into electrical energy or, conversely, electrical energy into mechanical energy. They have been extensively utilized as actuators, sensors, and transducers for various purposes.

The vibration control methodology can be catabolized into three types as follows: the active type, the passive type and the semi-active type. For active vibration suppression, a voltage or an electric charge has to be supplied from an external energy source to PZTs. It basically has the largest vibration suppression effect. However, for special structures that have limited energy sources, such as space structures and seabased platforms, it is imperative to minimize energy consumption. Moreover, since the accurate model of the large space structure is hard to be constructed due to the difficulty of preliminary experiments and the manufacturing error of the structure in the space, non-robust active vibration system might show the unstable behavior.

On the other hand, since passive method does not supply energy to the system, the passive system is always stable. A passive vibration control is easier to be implemented in actual systems than active vibration control system, because it does not require any controllers, sensors, or filters. However, in most cases, its 
vibration suppression effect is inferior to the one of the active control system and it might not provide enough performance.

The third type is the semi-active vibration control which dynamically changes the property of the structure according to the feedback of the state without adding extra energy. Semi-active systerns are usually able to achieve higher performance than pure passive methods without the disadvantages of active systems. Outlines of the various methods categorized into semi-active type can be found in comprehensive reviews $[3,4,5]$.

In this research, some works on semi-active vibration suppression having energy recycling effect with a circuit switching are focused $[6,7,8,9,10]$. In these methodologies, semi-active controllers composed of piezoelectric materials and switchable circuits whose ON-OFF is controlled according to the state of the controlled target. On the other hand, the design methodologies of control systems, such as the placement optimization of sensors and actuators, based on numerical techniques have long been studied (e.g. [11, 12, 13). The integrated optimization of the control system and the controlled structure also well studied (e.g. $[14,15,16,17])$. These methodologies could strongly contribute to the increase of the vibration suppression effect or the reduction of the devise cost. However, active or passive type methodologies are mainly treated in these optimizations. Thus the design methodology for the semi-active vibration suppression systems still has the room to be studied. Of course, the optimization methodology for switching based energy recycling method is still open problem. In this kind of control systems, the electric charge stored in the PZT could change drastically, the unique design methodology different from the one of active or passive can be required.

In this research, we construct the optimization methodology of the energy-recycling type semi-active vibration control system for a space structure proposed in [8]. First, the design variables are set as the length of the piezoelectric transducer (PZT) on each truss element. Physical properties of the actuator are formulated as the function of the design variables. Then, the vibration analysis problem of the truss structure is formulated based on the transient response analysis using the modal method. The objective function is set as the integration of the square of all displacement over the whole analysis time domain. The sensitivity of the objective function is derived based on the adjoint variable method $[18,19]$. Based on these formulations, an optimization algorithm is constructed using the method of moving asymptotes (MMA) [20]. Finally, we provide numerical examples to illustrate the validity and utility of the proposed methodology.

\section{Numerical modeling of energy-recycling semi-active vibration control systems}

\subsection{Formulation of piezoelectric transducer}

In this research, a space frame truss structure with some PZTs for vibration control is assumed as the design target as shown in Fig.1. First, the formulation of the element composed of the piezoelectric transducer on the truss structure is defined. In this research, let us consider the rod type PZT containing piezoceramic as shown in Fig.2. The PZT is connected to truss material and forms one rod element connecting two node of the structure. The integrated structure is modeled on having only axial stiffness and piezoelectric effect in the numerical analysis. Thus, piezoelectric effect of the PZT is formulated in the context of the truss element.

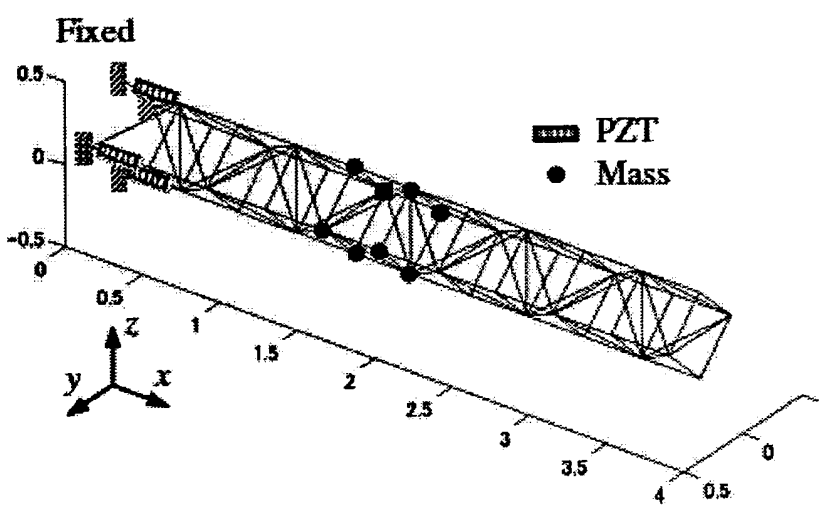

Figure 1: Vibration suppression target structure 


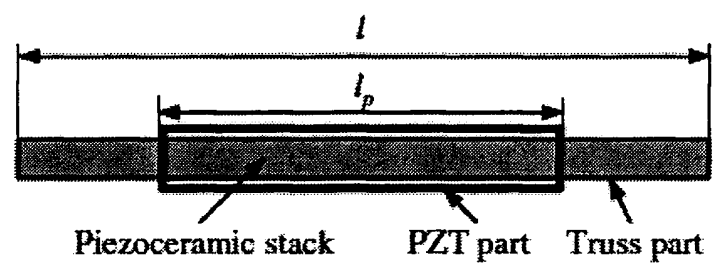

Figure 2: Outhe of piezoelectric transducer (PZT) connected with a truss

Let $l_{p}$ and $\delta l_{p}$ be the length and elongation of the PZT respectively, the equations of state of the PZT with stacked piezoceramics is formulated as follows [21]:

$$
\begin{aligned}
& f_{p}=k_{p} \delta l_{p}-b_{p} Q \\
& V=-b_{p} \delta l_{p}+\frac{Q}{C_{p}}
\end{aligned}
$$

where

$$
\begin{gathered}
k_{p}=\frac{A_{p} \epsilon_{33}}{l_{p}\left(\epsilon_{33} s_{33}-d_{33}{ }^{2}\right)} \\
b_{p}=\frac{d_{33}}{n_{p}\left(\epsilon_{33} s_{33}-d_{33}{ }^{2}\right)} \\
C_{p}=\frac{A_{p} n_{p}^{2}\left(\epsilon_{33} s_{33}-d_{33}{ }^{2}\right)}{l_{p} s_{33}}
\end{gathered}
$$

and $f_{p}$ is the axial force applying to the $\mathrm{PZT}, k_{p}, b_{p}, C_{p}, V, Q$ and $A_{p}$ are axial stiffness, piezoelectric coefficient, capacitance, voltage, electric charge and cross-sectional area of the PZT respectively, $s, d$ and $\epsilon$ are compliance, piezoelectricity and permittivity of the material of $\mathrm{PZT}, n_{p}$ is the number of the piezoceramic stacks.

Here we assume that the PZT and the truss element have the same axial stiffness for simplicity. Let $l$ and $\delta l$ be the length of the rod containing the PZT, the elongation of the PZT is formulated as follows:

$$
\delta l_{p}=\frac{l_{p}}{l} \delta l
$$

\subsection{Equations of state of truss structure with PZTs}

The structure is composed of $n_{e}$ linear truss elements having $n_{n}$ node points and $n_{p}$ PZTs. Let $m$ be the degrees of freedom of this model. Considering displacement vector $\mathbf{u}$ at the nodes and the electric charge vector $\mathbf{Q}$ at the PZTs as the state variables, the following equation of motion and piezoelectric equation are considered as the equations of state:

$$
\begin{gathered}
\mathbf{M} \ddot{\mathbf{u}}+\mathbf{C} \dot{\mathbf{u}}+\mathbf{K} \mathbf{u}=\mathbf{B}_{\mathbf{p}} \mathbf{Q} \\
\mathbf{V}=-\mathbf{B}_{\mathbf{p}}{ }^{T} \mathbf{u}+\mathbf{C}_{\mathbf{p}}^{-\mathbf{1}} \mathbf{Q}
\end{gathered}
$$

where $\mathbf{M}$ is a mass matrix, $\mathbf{C}$ is a damping matrix, $\mathbf{K}$ is a stiffness matrix, $\mathbf{B}_{\mathbf{p}}$ is a piezoelectric coupling matrix, $\mathbf{V}$ is a electric potential vector and $\mathbf{C}_{\mathbf{p}}$ is a capacitance matrix.

For the sake of the numerical effectiveness and vibration suppression based on the modal coordinate system, the displacement $\boldsymbol{u}$ in the global coordinates is transformed into the modal displacement $q$ as follows:

$$
\mathbf{u}=\phi \mathbf{q}
$$

where

$$
\phi=\left[\phi_{1}, \phi_{2}, \ldots, \phi_{m}\right]
$$


and $\phi_{k}$ is the $k-t h$ eigenvector of the following eigenvalue problern:

$$
\left(\mathbf{K}-\omega_{k}^{2} \mathbf{M}\right) \phi_{k}=0
$$

where

$$
\phi_{k}^{T} \mathbf{M} \phi_{k}=1
$$

and $\omega_{k}$ is $k$-th eigenfrequency. Substituting Eq.(9) into Eq. (7) and multiplying $\phi^{T}$ to it from the left, the following modal equation of state with the modal displacement $q$ is obtained.

$$
\ddot{\mathbf{q}}+2 \Xi \Omega^{\frac{1}{2}}+\mathbf{\Omega q}=\phi^{T} \mathbf{B}_{\mathbf{p}} \mathbf{Q}
$$

where

$$
\begin{gathered}
\boldsymbol{\Omega}=\operatorname{diagonal}\left[\omega_{1}^{2}, \omega_{2}^{2}, \ldots, \omega_{m}^{2}\right] \\
\boldsymbol{\Xi}=\operatorname{diagonal}\left[\xi_{1}, \xi_{2}, \ldots, \xi_{m}\right]
\end{gathered}
$$

\subsection{Energy-recycling serni-active vibration control}

The energy-recycling semi-active vibration control used for the control strategy of the vibration suppression of the structure [8] is outlined here. In this methodology, additional coil and switch are set for each PZT. Each PZT is modeled as series connection of a voltage source, a capacitor and a resistor. Thus each circuit is modeled as LCR-circuit with a switch as shown in Fig.3. Let $Q$ be the state variable, the state equation for all circuits is represented as follows for the two states, the switch is on and off:

$$
\begin{aligned}
& \text { When a swich is on : } \quad L_{i} \ddot{Q}_{i}+R_{i} \dot{Q}_{i}+C_{i}^{-1} Q_{i}=\left(\mathbf{B}_{\mathbf{p}}{ }^{T} \mathbf{u}\right)_{i} \\
& \text { When a swich is off : } \quad \dot{Q}_{i}=0
\end{aligned}
$$

where $L_{i}, R_{i}$ and $C_{i}$ are inductance, resistance and capacitance the circuit of $i$-th $P Z T,\left(B_{p}{ }^{T}\right)_{i}$ is axial elongation of -th PZT actuator. Here, we assume that the resistance of PZT is dominant in the circuit and the resistance of the coil can be ignored. The switch is operated to achieve the feedback control for vibration suppression partially based on the active control theory. The electric charge is stored to the PZT effectively by changing the polarity of the charge. Charging polarity is controlled by the switches of circuits of each PZT. Let us the target electric charge of the $i$-th PZT be $Q_{T i}$ in this semi-active control, the operating low for the switch of the circuit for $i$-th piezoelectric actuator is formulated as follows:

$$
\text { When } Q_{T i} V_{i}<0 \text { turn on the switch for a duration of } \pi / \omega_{c i}
$$

where $\omega_{c j}$ is an eigenfrequency of the circuit of $i$-th PZT. The strategy of this methodology is to control the switch of the circuit so that $Q_{i}$ has the same sign as $Q_{T_{i}}$ and the absolute value of $Q_{i}$ is as large as possible. After the switch is turned on, $Q_{i}$ begins to decrease when $V_{i}$ is positive, while $Q_{i}$ begins to increase when $V_{\hat{k}}$ is negative. However, the decrease (or increase) in electric charge $Q_{i}$ does not continue for long due to electric oscillation. Assuming that the electric oscillation is much faster than the structural one, the variation of electric charge can be fixed to its maximum value if the switch is turned at the half period of the electric oscillation. Figure 4 shows the relationship between the displacement and the electric charge when the simusoidal displacement is forced to the actuator and the target electric charge is set according to direct velocity feedback, that is, $Q_{T i}=-\alpha \dot{d}_{i}$, where $\alpha$ is an arbitrary positive gain. 


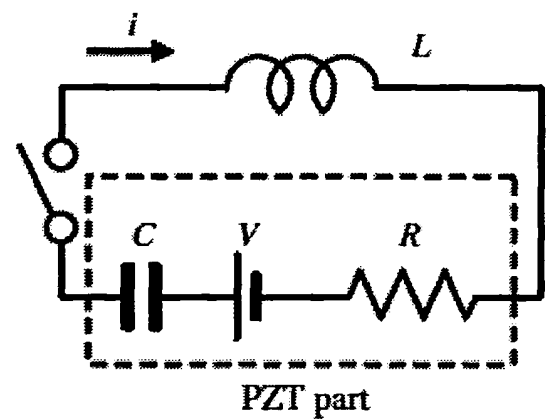

Figure 3: Outline of LCR circuit of each PZT

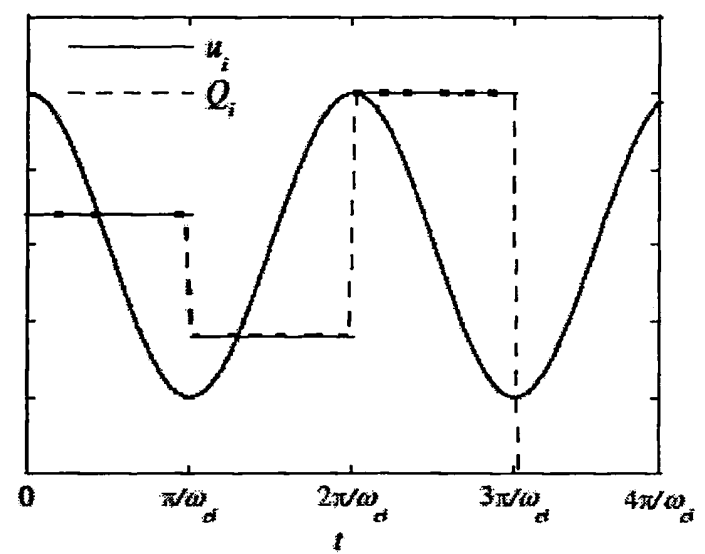

Figure 4: Outline of variation of displacement and electric charge in energy recycling semi-active vibration control

In the multi-degrees of freedorn system, $Q_{T i}$ is decided according to the linear quadratic regulator (LQR) control theory. The state equations in modal coordinate is rewritten in as follows considering electric charge $Q$ as control variable $Q_{T}$ :

$$
\dot{\mathbf{z}}=\mathbf{A z}+\mathbf{B Q} \mathbf{T}
$$

where

$$
\begin{gathered}
\mathbf{z}=\left[\begin{array}{cc}
\mathbf{0} & \mathbf{I} \\
-\mathbf{\Omega} & -2 \Xi \mathbf{\Omega}^{\frac{1}{2}}
\end{array}\right] \\
\boldsymbol{A}=\left[\begin{array}{c}
\mathbf{q} \\
\dot{\mathbf{q}}
\end{array}\right] \\
\boldsymbol{B}=\left[\begin{array}{c}
\mathbf{0} \\
\phi^{T} \mathbf{B}_{\mathbf{p}}
\end{array}\right] \\
\mathbf{Q}_{\mathbf{T}}=\left[Q_{T 1}, Q_{T \mathbf{2}}, \ldots, Q_{T n_{p}}\right]^{T}
\end{gathered}
$$

That is, the optimal $Q_{\mathbf{T}}$ is obtained as the minimizer of the following cost function.

$$
J=\int \mathbf{z}^{T} \mathbf{W}_{\mathbf{1}} \mathbf{z}+\mathbf{Q}_{\mathbf{T}}{ }^{T} \mathbf{W}_{2} \mathbf{Q}_{\mathbf{T}} \mathrm{d} t
$$

Using the positive definite solution $\mathbf{P}$ of the following Riccati equation:

$$
\mathbf{P B}_{\mathbf{p}} \mathbf{W}_{2}^{-1} \mathbf{P}-\mathbf{A}^{T} \mathbf{P}-\mathbf{P A}-\mathbf{W}_{1}=0
$$

$Q_{\mathbf{T}}$ is calculated as follows:

$$
\mathbf{Q}_{\mathbf{T}}=-\mathbf{F} \mathbf{z}
$$


where

$$
\mathbf{F}=\mathbf{W}_{3}^{-1} \mathbf{B}_{\mathbf{p}}{ }^{T} \mathbf{P}
$$

\section{Optimization methodology}

3.1 Design variables

In this research, we intend to obtain the optimal layout and length of piezoelectric actuators. Based on the concept of ground structure approach [22] of truss structure, the candidates of the layout position are set on all truss elements constructing the target structure. That is, $n_{p}$ equals to $n_{e}$ here. The lengths of the PZT on each truss are considered as design variables. Very small design variables represent non-existence of PZT in this method. The following length ratio vector between total bar length and the PZT $r$ is regarded as design variable.

$$
\mathbf{r}=\left[r_{1}, r_{2}, \ldots, r_{n_{p}}\right]
$$

where

$$
\begin{gathered}
r_{i}=\frac{l_{p i}}{l_{i}} \\
r_{\min } \leq r_{i} \leq 1
\end{gathered}
$$

and $r_{\min }$ is the minimum value of $r_{i}$. The piezoelectricity $b_{p i}$, capacitance $C_{p i}$ and resistance $R_{i}$ in Eqs.(13) and (16) depend on the length of the PZT. Assuming that the number of stacks $n_{p i}$ of the $i$-th PZT is linear contimuous function of $r_{i}$ as follows:

$$
n_{p i} \approx n_{\max i} * r_{i}
$$

where $n_{\max i}$ is the number of the stacks when $r_{i}=1$, they are represented as the function of $r_{i}$ :

$$
\begin{gathered}
b_{p i}=\frac{b_{0}}{n_{p i}} \approx \frac{b_{0}}{n_{\max i}} r_{i}^{-1} \\
C_{p i}=\frac{A_{p i} n_{p i}^{2} C_{0}}{l_{p i}} \approx \frac{A_{p i} n_{\max i}^{2} C_{0}}{l_{i}} r_{i} \\
R_{p i}=\frac{R_{0}}{n_{p i}}=\frac{R_{0}}{n_{\max i}} r_{i}^{-1}
\end{gathered}
$$

where

$$
\begin{aligned}
b_{0} & =\frac{d_{33}}{\epsilon_{33} s_{33}-d_{33}{ }^{2}} \\
C_{0} & =\frac{\epsilon_{33} s_{33}-d_{33}{ }^{2}}{s_{33}}
\end{aligned}
$$

and $R_{0}$ is the resistance of a piece of piezocelamic constructing stacks.

Note that, the Riccati equation in Eq.(25) contains the piezoelectric matrix $B_{p}$, the coefficient matrix $F$ of the target value of electric charge can vary during optimization. However, for simplicity, the weighting matrix $W_{2}$ is set to make the Ricatti equation independent of the design variable as follows:

$$
\mathbf{W}_{2}=\operatorname{diagonal}[\mathbf{r}]
$$

According to this setting, the Ricatti equation and the solution of it don't vary during optimization.

3.2 Optimization problem

As the objective function $F$ of this optimization problem, the time integration of the square norm of the displacement vector is used as follows.

$$
F(\mathbf{r}, \mathbf{u}, \mathbf{Q})=\int_{0}^{T}\|\mathbf{u}\|^{2} \mathrm{~d} t
$$


where $T$ is the end time of evaluation. As discussed the former subsection, positive correlation is observed between the PZT length and the vibration suppression effect. Thus, the optimal solution could have all possible PZTs with their maximum length. To suppress the total length of PZT, the length constraint is here introduced. The optimization problem is represented as follows:

$$
\underset{\mathbf{r}}{\operatorname{minimize}} F(\mathbf{r}, \mathbf{u}, \mathbf{Q})=\int_{0}^{T^{*}}\|\mathbf{u}\|^{2} \mathrm{~d} t
$$

Subject to

$$
\begin{gathered}
\sum_{i=1}^{n_{p}} r_{i} l_{p} \leq l^{U} \\
r_{\min } \leq r_{i} \leq 1 \text { for } i=1, \ldots, n_{p}
\end{gathered}
$$

and $l^{U}$ is the upper bound for the total length of $\mathrm{PZT}$.

\subsection{Sensitivity analysis}

Since the design variables are updated using MMA, we requires the first order sensitivity of the objective function and constraints to perform the optimization. Since the derivation is lengthy, only the results are shown here and the detailed derivation is shown in the Appendix. Using the adjoint variable method [18, 19], the derivative of the objective function with respect to $i$-th design variable $r_{i}$ is formulated as a function of the state variable $\mathbf{Q}$ and the adjoint variables $\lambda$ and $\mu$ :

$$
\frac{d F}{d r_{i}}=-\int_{0}^{T} \lambda^{T} \frac{\partial \mathbf{B}_{\mathbf{p}}}{\partial r_{i}} \mathrm{Qd} t+\int_{T_{S i}} \mu_{i}\left\{\frac{\partial}{\partial r_{i}}\left(\frac{R_{i}}{L_{i}}\right) \dot{Q}_{i}+\frac{\partial}{\partial r_{i}}\left(\frac{1}{R_{i} L_{i}}\right) Q_{i}\right\} \mathrm{d} t
$$

and $T_{S i}$ is the time when $i-t h$ swich is on. The adjoint variables are obtained by solving following adjoint equations:

$$
\begin{gathered}
\qquad \hat{\eta}+2 \Xi \Omega^{\frac{1}{2}} \dot{\eta}+\Omega \eta=\phi^{T}\left(\mathbf{B}_{\mathbf{p}} \mu_{\mathrm{s}}-2 \mathbf{u}\right) \\
\text { When the swich of } i \text {-th circuit is on : } \ddot{\mu}_{i}-\frac{R_{i}}{L_{i}} \mu_{i}+\frac{1}{L_{i} C_{i}} \mu_{i}=-\left(\mathbf{B}_{\mathbf{p}} \lambda\right)_{i} \\
\text { When the swich of } i \text {-th circuit is off : } \mu_{i}=\left(\mathbf{B}_{\mathbf{p}} \lambda\right)_{i}
\end{gathered}
$$

where

$$
\begin{aligned}
& \mu_{\mathrm{s}}=\left[\mu_{a 1}, \mu_{a 2}, \ldots, \mu_{a n_{p}}\right]^{T}, \quad \mu_{s i}=\left\{\begin{array}{l}
\text { When the swich of } i \text {-th circuit is on }: \mu_{i} \\
\text { When the swich of } i \text {-th circuit is off }: 0
\end{array}\right. \\
& \lambda=\phi \eta
\end{aligned}
$$

Terminal conditions :

$$
\begin{aligned}
& \boldsymbol{\eta}(T)=\mathbf{0} \\
& \dot{\eta}(T)=\mathbf{0} \\
& \boldsymbol{\mu}(T)=\mathbf{0} \\
& \dot{\mu}(T)=\mathbf{0}
\end{aligned}
$$

where $\left(\mathrm{B}_{\mathrm{p}} \lambda\right)_{i}$ is the virtual elongation of $i$-th $\mathrm{PZT}$ calculated from adjoint variable $\lambda$ in global coordinate system.

\subsection{Algorithm}

The optimization algorithm is constructed based on the above formulations. The eigenvalues and eigenvectors of the system in Eq.(11) and the feed-back matrix of LQR control theory in Eq.(27) are calculated first since they do not vary during optimization. In the optimization loop, the state equations and adjoint equations are solved by the forth order Runge-Kutta method. The state equations are an initial value problem, while the adjoint equations are a terminal value problem. The design variable are updated by the method of moving 
asymptotes (MMA) [20] using the calculated sensitivity.

\section{Numerical example}

The following numerical examples are provided to confirm the validity and the utility of the proposed methodology using the truss structure shown in Fig.1. In all examples, aluminum truss element with the stiffness $1.99 \times 10^{6} \mathrm{~N}$ and the length density $9.39 \times 10^{-2} \mathrm{~N} / \mathrm{m}$ is used. For the simplicity, the piezoelectric actuator is assumed that having the same stiffness and density. Other properties of PZT in Eqs.(32)-(34) are set as follows, $b_{0}=1.31 \times 10^{8} \mathrm{~N} / \mathrm{C}, A_{\mathrm{pi}}=2.88 \times 10^{-5} \mathrm{~m}^{2}, C_{0}=2.28 \times 10^{-7} \mathrm{~F}, R_{0}=1.97 \times 10^{3} \Omega$ and $n_{\max }=1300$. The length of each bay is set as $0.38 \mathrm{~m}$. $0.5 \mathrm{~kg}$ masses are mounted at all nodes of the 3-rd and 4-th bay. Above all physical factors are based on the experimental data shown in [23].

All eigenmodes are used to perform calculation in the modal coordinate system. During the optimization, the PZT are set to all truss elements. For simplicity, the maximum length of PZT is set to $0.38 \mathrm{~m}$ which equals to the length of the bay. The timestep for the integration is set to $1.0 \times 10^{-5} \mathrm{~s}$, which is enough shorter than the period of the highest order vibration of the structure $\left(4.88 \times 10^{-4} \mathrm{~s}\right)$. The upper bound for the sum of design variable in Eq.(40) is set to 13 which corresponds to $10 \%$ of maximum total length of PZTs.

As the first benchmark example, optimization of the PZT layout and length for the first mode dominant free vibration is performed. The initial modal velocity is set to $0.1 \mathrm{mkg}^{\frac{1}{2}}$ to the first mode and 0 to the other modes as the same with the previous. The weighting matrix $W_{1}$ of LQR control theory in Eq.(24) is set to $\mathrm{W}_{1}=$ diagonal $\left[1,1 / \omega_{1}^{2}\right]$. Figure 5 shows the optimal $r$ of each PZT after 100 optimization iterations. The optimal length of each PZT is represented by the color of bars. Base truss structure is plotted by chain lines. Figure 6 shows convergence history of the objective function. Long PZTs are located on the upper and the lower side of the structure and the length constraint became active. Since the highest axial force yield on the upper and the lower side of the structure in the first order vibration, this layout is mechanically reasonable. Moreover, the stable convergence is observed in Fig.6. It shows that the proposed optimization procedure correctly worked in this example. Figure 7 shows the variation of electric charge of the PZT located on the upper left side and the first order modal displacement of the optimal structure. Since the switching is performed near the peak of the displacement and the electric charge is efficiently stored to PZT, the energy recycling semi-active vibration control certainly worked well in this example.

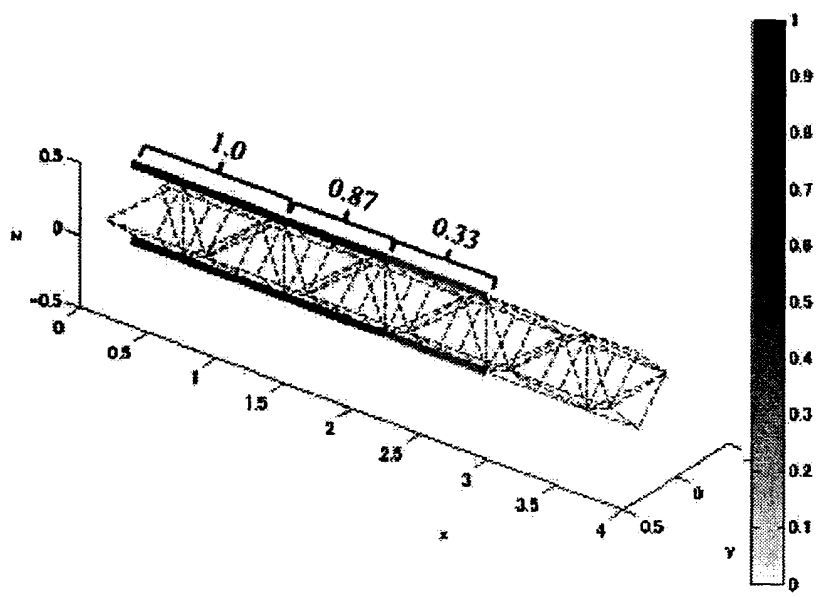

Figure 5: Optimal layout of PZT for the first mode dominant vibration 


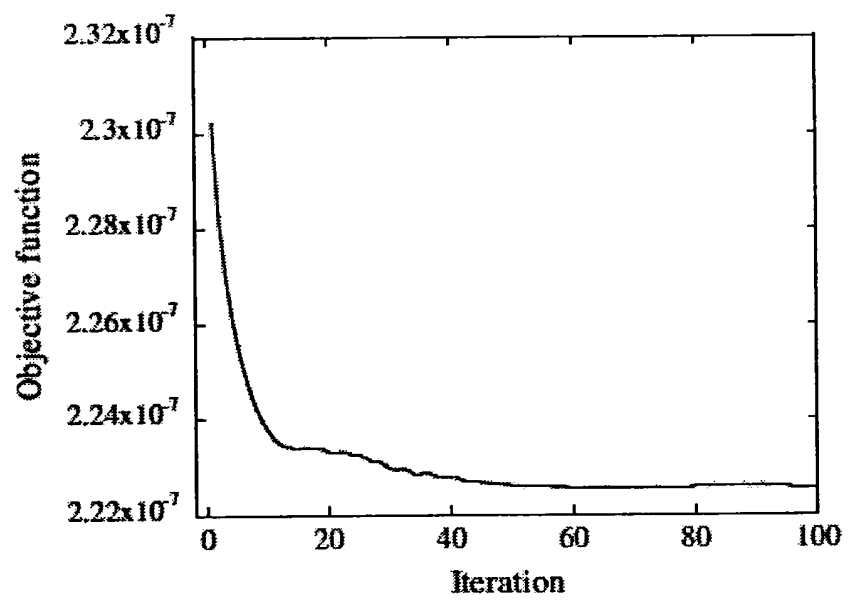

Figure 6: History of optimization for the first mode dominant vibration

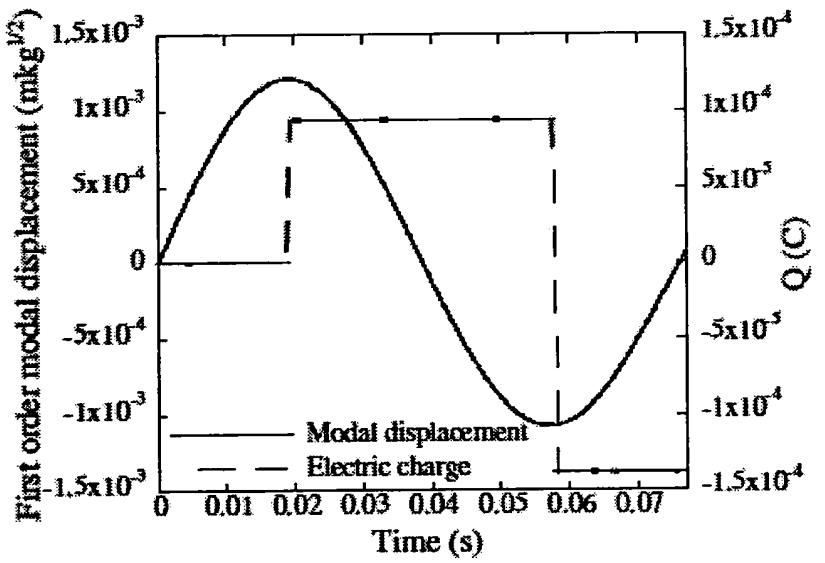

Figure 7: The history of the first order modal displacernent and electric charge stored in a PZT

\section{Conclusion}

In this research, we constructed the optimization methodology of the energy-recycling type semi-active vibration control system for a space structure. The design variables were set as the length of the piezoelectric actuator on each truss element. Physical properties of the actuator were formulated as the function of the design variables. The vibration analysis problem of the truss structure was formulated based on the transient response analysis using the modal method. The objective function was set as the integration of the square of all displacement over the whole analysis time domain. The sensitivity of the objective function was derived based on the adjoint variable method. Based on these formulations, an optimization algorithm was constructed using the method of moving asymptotes (MMA). Numerical examples were provided to demonstrate the validity of our method.

\section{References}

[1] G. A. Lesieutre. Vibration damping and control using shunted piezoelectric materials. Shock Vib. Digest, 30(3):187-195, 1998.

[2] M. Ahmadian and A. P. Deguilio. Recent advances in the use of piezoceramics for vibration suppression. Shock Vib. Digest, 33(1):15-22, 2001.

[3] D. Karnopp. Active and semi-active vibration isolation. J. Mech. Des., 117:177-185, 1995. 
[4] M. D. Symans and M. C. Constantinou. Semi-active control systems for seismic protection of structures: a state-of-the-art review. Eng. Struct., 21(6):469-487, 1999.

[5] N. Jahli. A comparative study and analysis of semi-active vibration-control systems. J. Vib. Acoust.* $124(4): 593-605,2002$.

[6] C. Richard, D. Guyomar, D. Audigier, and H. Bassaler. Enhanced semi-passive damping using continuous switching of a piezoelectric device on an inductor. In Proceedings of SPIE, volume 3989, pages 288-299, 2000.

[7] L. R. Corr and W. W. Clark. A novel semi-active multi-modal vibration control law for a piezoceramic actuator. J. Vib. Acoust., 125:214-222, 2003.

[8] J. Onoda, K. Makihara, and K. Minesugi. Energy-recycling semi-active method for vibration suppression with piezoelectric transducers. ALAA journal, 41(4):711-719, 2003.

[9] K. Makihara, J. Onoda, and K. Minesugi. Comprehensive assessment of semi-active vibration suppression including energy analysis. $J$. Vib. Acoust., 129:84-93, 2007.

[10] J. Qiu, H. Ji, and K. Zhu. Semi-active vibration control using piezoelectric actuators in smart structures. Front Mech. Eng. China, 4(3):242-251, 2009.

[11] G. Schulz and G. Heimbold. Dislocated actuator/sensor positioning and feedback design for flexible structures. J. Guid. Contr. Dynam, 6:361-367, 1983.

[12] R. E. Lindberg. On the number and placement of actuators for independent modal space control (for large flexible spacecraft). J. Guid. Contr. Dynam., 7:215-221, 1984.

[13] A. Hac and L. Liu. Sensor and actuator location in motion control of flexible structures. J. Sound Vib., 167(2):239-61, 1993

[14] A. L. Hale, W. E. Dahl, and R. J. Lisowiki. Optimal simultaneous structural and control design of maneuvering flexible spacecraft. J. Guid. Contr. Dynam, 1985.

[15] J. Onoda and R. T. Haftka. An approach to structure/control simultaneous optimization for large flexible spacecraft. AIAA Joumal, 25(8):1133-1138, 1987.

[16] I. Kajiwara, K. Tsujioka, and A. Nagamatsu. Approach for simultaneous optimization of a structure and control system. AIAA Jounal, 32(4):866-873, 1994.

[17] J. S. Ou and N. Kikuchi. Integrated optimal structural and vibration control design. Struct. Optim., 12(4):209-216, 1996.

[18] E. J. Haug, K. K. Choi, and V. Komkov. Design Sensitivity Analysis of Structural Systems. Academic Press, Orlando, FL, 1986.

[19] B. S. Kang, G. J. Park, and J. S. Arora. A review of optimization of structures subjected to transient loads. Structural and Multidisciplinary Optimization, 31(2):81-95, 2006.

[20] K. Svanberg. The method of moving asymptotes- a new method for structural optimization. Int. J. Numer. Meth. Eng., 24(2):359-373, 1987.

[21] M. Nelkon. Electricity and Magnetism. Edward Arnold, second edition, 1965.

[22] M. P. Bendsøe and O. Sigmund. Topology Optimization: Theory, Methods, and Applications. SpringerVerlag, Berlin, 2003.

[23] K. Makihara. Energy-recycling Semi-active Vibration Suppression of Space Structures. PhD thesis, The University of Tokyo, 2003. 